\section{Acetazolamide and SGLT2 inhibitor as potent drugs for a patient with diabetes mellitus and worsening chronic lym- phedema: A case report}

\author{
Hajime Kataoka \\ Division of Internal Medicine, Nishida \\ Hospital, Saiki-city, Oita, Japan
}

\begin{abstract}
Treatment of lymphedema using a pharmacologic approach is reported to have limited efficacy. Here, I report a patient with type 2 diabetes (T2DM) and acute worsening of her chronic lymphedema, in whom treatment with acetazolamide and a sodiumglucose cotransporter-2 inhibitor (SGLT2i) effectively improved the lymphedema. A 94-year-old woman, who was treated for T2DM, hyperlipidemia, and hypertension for 17 years at my hospital presented to the emergency room because of acute worsening of her chronic right leg lymphedema with increased swelling, tightness, and dull aching. A pharmacologic approach was used to treat her worsening lymphedema. Acetazolamide $500 \mathrm{mg} / \mathrm{d}$ was administered to treat the acute tissue fluid collection in the right lymphedematous leg. Ten days later, the patient's body weight was markedly reduced by $3.2 \mathrm{~kg}$, pitting in the right leg was markedly improved, and the circumference of right limb was decreased. On day 11 , the glucose-lowering agent vildagliptin was switched to the SGLT2i empagliflozin $10 \mathrm{mg} / \mathrm{d}$. On day 70, her body weight had decreased further by $2.8 \mathrm{~kg}$, and the circumference of her right limb was greatly reduced compared with that under treatment with acetazolamide. Her serum chloride concentration was increased after treatment, but her hemoglobin and hematocrit values did not change during the study period. In conclusion, acetazolamide and an SGLT2 $\mathrm{i}$ have acute diuretic effects for draining the excess tissue fluid in the lymphedematous limb without vascular contraction by enhancing vascular tonicity. Additionally, an SGLT2i may have chronic effects for reducing fat deposits in the lymphedematous limb.
\end{abstract}

\section{Introduction}

The lymphatic system drains through the lymph nodes and lymphedema generally arises due to an obstruction in the lymphatic system, mainly due to surgery, radiation or trauma. ${ }^{1}$ In particular, lymphedema is an important consideration for clinicians who care for cancer patients because of its relatively high frequency and significant functional and quality of life implications for patients. ${ }^{1}$ Diuretic treatment of lymphedema is reported to have limited efficacy because it does not eliminate the protein that attracts and hangs onto the extra lymph fluid. ${ }^{1}$ The chloride theory for heart failure pathophysiology, ${ }^{2}$ however, promotes the active use of acetazolamide in cardiovascular patients. ${ }^{3,4}$ The chloride theory suggests that acetazolamide could theoretically improve lymphedema by its pharmacologic action to enhance vascular tonicity by elevating serum chloride concentration, ${ }^{5}$ which might facilitate the drainage of excessive interstitial lymphatic fluid into the vascular space of venous vessels, and reduce lymphedema without vascular contraction. ${ }^{6}$

The newly developed glucose-lowering drugs, sodium-glucose cotransporter-2 inhibitors (SGLT2i), have similar diuretic effects as acetazolamide in that they have a chloride-regaining diuretic action..$^{7,8}$ An SGLT2i may also improve lymphedema through the reduction of adipose tissue deposits in lymphedema. ${ }^{9,10}$ by its metabolic effect to reduce the body fat mass. ${ }^{11,12}$ Effects of acetazolamide and an SGLT2 inhibitor on lymphedema are unknown yet. Here, I report a patient with type 2 diabetes (T2DM) and acute worsening of her chronic lymphedema, in whom treatment with acetazolamide and an SGLT2i effectively improved the lymphedema.

\section{Case Report}

A 94-year-old woman, treated for T2DM, hyperlipidemia, and hypertension for 17 years at my hospital, presented to the emergency room due to acute worsening of her chronic right leg lymphedema with swelling, tightness, and dull aching. The etiology of her lymphedema was considered to be a gynecologic surgery performed when she was young. She had not received previous treatment for her lymphedema, except for trying the use of a compression stocking 7 years earlier, which failed to provide relief. She had no previous episodes of heart failure or worsening renal function. She was being treated at the time with a daily dose of the glucose-lowering agent vildagliptin (50 $\mathrm{mg})$; a lipid-lowering agent, pravastatin (10 $\mathrm{mg}$ ); and antihypertensive agents, efonidipine $(20 \mathrm{mg})$ and irbesartan $(100 \mathrm{mg})$.

She was admitted to the hospital for acute worsening of her chronic lymphede-
Correspondence: Hajime Kataoka, Department of Internal Medicine, Nishida Hospital, Tsuruoka-Nishi-machi 2-266, SaikiCity, Oita 876-0047, Japan.

Tel.: +81.972220180 - Fax: +81.972233053 E-mail: hkata@cream.plala.or.jp

Key words: Case report; lymphedema; acetazolamide; SGLT2 inhibitor; fat deposit.

Conflict of interest: The author declares no conflict of interest.

Ethics: This case report was approved by the ethics committee of Nishida Hospital.

Informed consent: Informed consent was obtained from the patient and her family members before study enrollment.

Received for publication: 8 February 2020 .

Revision received: 7 April 2020.

Accepted for publication: 18 April 2020.

This work is licensed under a Creative Commons Attribution 4.0 License (by-nc 4.0).

${ }^{\circ}$ Copyright: the Author(s), 2020

Licensee PAGEPress, Italy

Veins and Lymphatics 2020; 9:8889

doi:10.4081/vl.2020.8889

ma. Table 1 shows the changes in physical and various clinical tests before and after the administration of acetazolamide (Diamox $500 \mathrm{mg} / \mathrm{d}$ ) during her 10-day hospital stay, followed by add-on SGLT2 $\mathrm{i}$ (empagliflozin $10 \mathrm{mg} / \mathrm{d}$ ) treatment at the outpatient clinic. Laboratory tests included peripheral blood and blood chemical tests, measurement of b-type natriuretic peptide, and DM/lipid-related tests. Changes in the circumference of both lower legs were measured at three fixed levels in each leg (10 $\mathrm{cm}$ above and below the knee, and around the ankle). The background use of antihypertensive agents and the lipid-lowering agent described above was kept constant throughout the study period, but the glucose-lowering drug vildagliptin was discontinued immediately after initiating treatment with the SGLT2i empagliflozin.

At baseline (day 0; Table 1), her habitus was $146 \mathrm{~cm}$ tall and weighing $64.1 \mathrm{~kg}$ (body mass index of 32.2). Her blood pressure was $152 / 82 \mathrm{mmHg}$, and she had a regular heart rate 64 beats $/ \mathrm{min}$, temperature of $36.5^{\circ} \mathrm{C}$, and oxygen saturation of $96 \%$ on ambient air. Physical examination showed no HF-related physical signs of neck vein distension, extra-cardiac sounds, and pulmonary rales, but moderate swelling of her right leg edema with mild pitting. Unilateral swelling in the whole right leg was apparent 
on inspection (Figure 1A), and physical examination revealed thickened and hyperkeratotic skin with mild pitting and normal coloring, and without reddish skin area, varicose veins, or venous ulceration. Cardiac examination showed a preserved left ventricular ejection fraction $(56 \%)$ and non-dilated diastolic volume $(89 \mathrm{cc})$ on cardiac ultrasound, with a near normal serum b-type natriuretic peptide level (48.6 $\mathrm{pg} / \mathrm{mL}$ ). Ultrasound examination, including duplex ultrasonography, revealed no anatomic or functional abnormalities in the inferior vena cava and venous system in the right lower leg, including deep venous thrombosis. Acute cellulitis was ruled out by the absence of an inflammatory reaction (white blood cell count, $5.49 \times 10^{3}$ cells $/ \mu \mathrm{L}$; C-reactive protein, $0.07 \mathrm{mg} / \mathrm{dL}$ ), and by consultation with the dermatologist at my hospital. Thus, the etiology of the unilateral swelling in the right leg was diagnosed as acute worsening of chronic lymphedema. ${ }^{13}$ The lymphedema in this patient was classified as late stage II because of the presence of mild pitting edema and presumed excess fat deposition in the diseased limb. ${ }^{1}$

Upon admission to the hospital, a pharmacologic approach was used to treat the acute worsening of her chronic lymphedema. Acetazolamide was administered at a daily dose of $500 \mathrm{mg}$ to treat the acute tissue fluid collection in the right lymphedematous limb. Ten days later (day 10, Table 1), the patient's body weight was markedly reduced by $3.2 \mathrm{~kg}$, pitting in the right limb was markedly improved, and the circumference of right limb in each level decreased by a maximum of $12.3 \%$ at the level $10 \mathrm{~cm}$ below the knee, compared with baseline (Figure 1B). Changes in the hemoglobin ($0.2 \mathrm{~g} / \mathrm{dL})$ and hematocrit $(-0.3 \%)$ values from baseline were mild despite the marked diuresis, indicating less vascular contraction after acetazolamide administration. The serum chloride concentration was increased from $105 \mathrm{mEq} / \mathrm{L}$ at baseline to $111 \mathrm{mEq} / \mathrm{L}$ after treatment.

Table 1. Changes in physical and blood tests after drug treatment for lymphedema in the right lower limb.

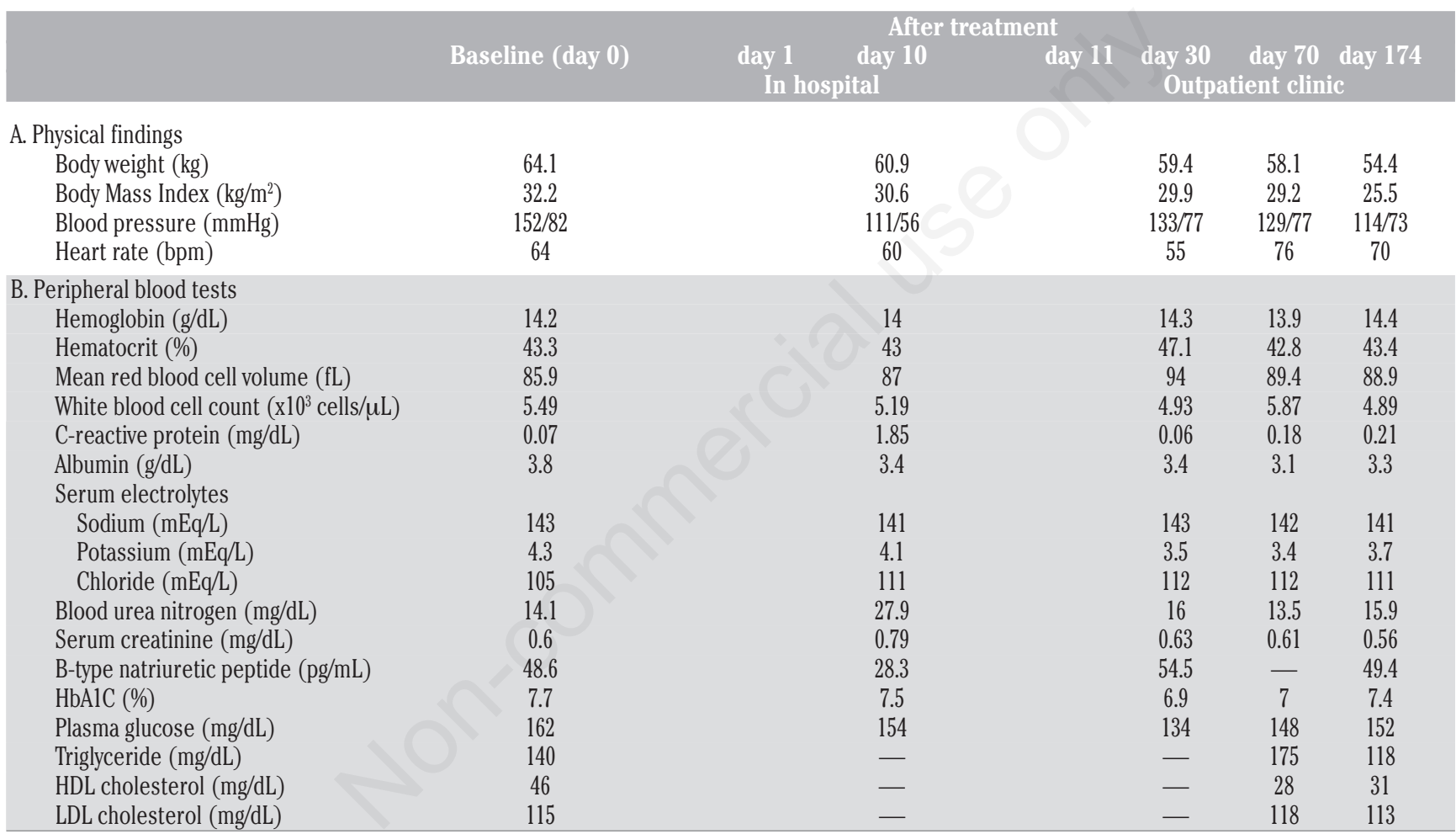

C. Measurement of lower limb circumference

Right lower limb

$10 \mathrm{~cm}$ above the knee $(\mathrm{cm})$ Percent change (\%)

$10 \mathrm{~cm}$ below the knee $(\mathrm{cm})$ Percent change (\%)

Ankle $(\mathrm{cm})$ Percent change (\%)

Left lower limb

$10 \mathrm{~cm}$ above the knee $(\mathrm{cm})$ Percent change (\%)

$10 \mathrm{~cm}$ below the knee $(\mathrm{cm})$ Percent change (\%)

Ankle $(\mathrm{cm})$ Percent change (\%)

$\begin{array}{lc}47.6 & 43.2 \\ \overline{44} & -9.2 \\ \overline{29} & 38.6 \\ - & -12.3 \\ & 28 \\ 43.4 & -3.4 \\ \overline{32.4} & \\ \overline{22.8} & 42 \\ - & -3.2 \\ & 32.8 \\ & 1.2 \\ & 22.6 \\ \end{array}$

$\begin{array}{ccc}43 & 41.4 & 40.5 \\ -9.7 & -13 & -14.9 \\ 40 & 34.6 & 35.3 \\ -9.1 & -21.4 & -19.8 \\ 28 & 23.7 & 23.2 \\ -3.4 & -18.3 & -20 \\ & & \\ 40.3 & 39.5 & 39 \\ -7.1 & -9 & -10 \\ 32 & 30 & 30.7 \\ -1.2 & -7.4 & -5.2 \\ 23.3 & 21.4 & 22 \\ 2.2 & -6.1 & -3.5\end{array}$

D. Treatment (daily dose)

Acetazolamide SGLT2 inhibitor

Diamox 500mg Empagliflozin 10mg

Percent change $=$ degree of change (\%) from baseline. 
Her lymphedematous condition greatly improved after the 10-day in-hospital treatment, and she was therefore discharged with continuing treatment at the outpatient clinic. On day 11 (Table 1), treatment for type 2 diabetes was switched from vildagliptin to the SGLT2i empagliflozin $(10 \mathrm{mg} / \mathrm{d})$ because of her high HbA1c level $(7.5 \%)$ and an expectation for further improvement in the right lymphedematous limb by the chloride-regaining diuretic effect of empagliflozin. ${ }^{7,8}$ Twenty days later (day 30, Table 1), her body weight was slightly reduced by $1.5 \mathrm{~kg}$ and the pitting in the right leg had disappeared almost completely, but the circumference of the right limb at each level had not changed. On day 70 (Table 1), however, her body weight decreased further by $1.3 \mathrm{~kg}$, and the circumference of the right limb was greatly decreased by a maximum of $21.4 \%$ at the level of $10 \mathrm{~cm}$ below the knee compared with that at baseline (Figure 1C). Her serum chloride concentration was preserved, and her hemoglobin and hematocrit values were not changed on days 30 and 70 . The HbA1c level was reduced around $7 \%$ on days 30 and 70 after treatment. As for the healthy left limb, the circumference at each level gradually and mildly decreased throughout the study period by a maximum of $9 \%$ at the level of $10 \mathrm{~cm}$ above the knee on day 70 compared with that at baseline, remaining stable at 6 months (day 174) after the initiation of pharmacologic treatment for acute worsening of her chronic lymphedema (Table 1). This case report was approved by the ethics committee of my hospital. Informed consent was obtained from the patient and her family members before study enrollment.

\section{Discussion}

To the best of my knowledge, this is the first reported case for which a potent pharmacologic approach with a combination of acetazolamide and SGLT2i was used to treat chronic or acute lymphedema. Both acetazolamide and SGLT2i may have acute diuretic effects for draining excess tissue fluid in the lymphedematous limb without vascular contraction. In addition to this diuretic effect, the SGLT2i may have chronic effects to reduce fat tissue deposits in lymphedematous limbs. ${ }^{9,10}$ The possible mechanisms of these drugs for improving the background pathophysiologic conditions in lymphedema are discussed below.

\section{Drainage of excess tissue fluid in the lymphedematous limb}

In lymphedema, drainage of excess tissue fluid in diseased limbs is compromised by obstruction/disruption of the lympho-vascular system due to primary or secondary etiologies. ${ }^{1}$ The use of diuretics is not considered effective or physiologically sound for improving lymphedema, ${ }^{1}$ but this report suggests that the use of acetazolamide and possibly SGLT $2 \mathrm{i}$ is useful for acute or chronic swelling of lymphedematous limbs because these diuretics can drain excessive interstitial lymphatic fluid into the vascular space without vascular contraction $^{8,14,15}$ through their pharmacologic actions to enhance vascular tonicity. ${ }^{2,5}$ Such a pharmacologic effect of draining the tissue fluid into the vascular space may be analogous to the effects caused by standard physical therapy using manual massage, compressive bandages, and pneumatic compression of the lymphedematous limb. ${ }^{1}$
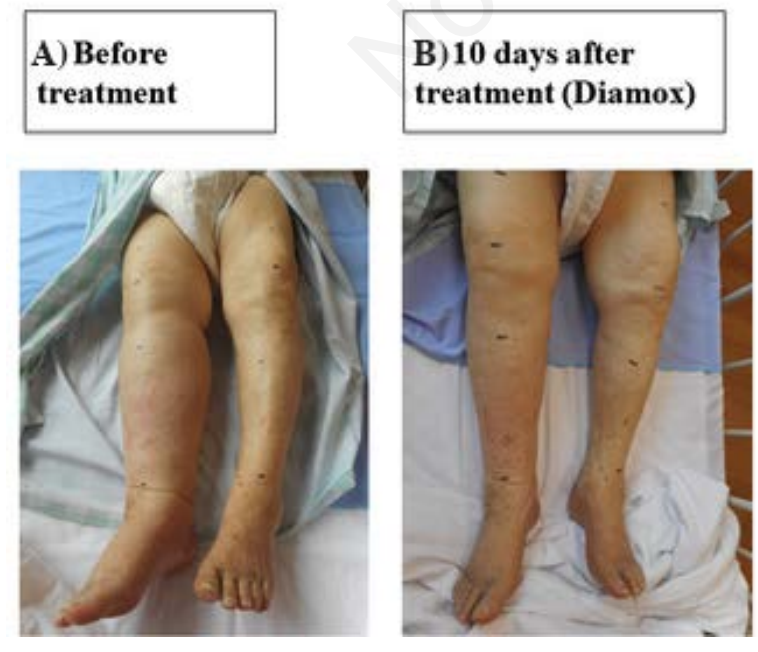
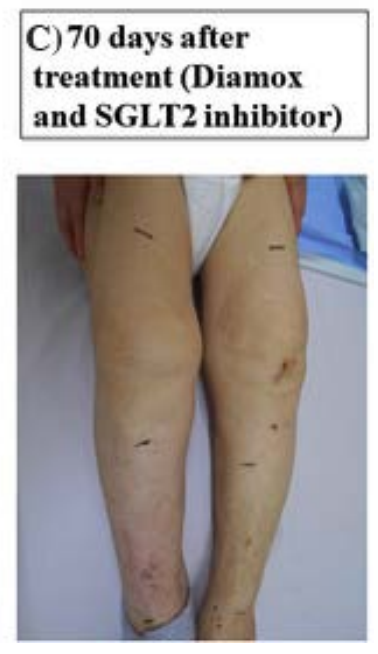

Figure 1. Serial changes in the right lower lymphedematous limb and left healthy limb at baseline (A), after acetazolamide (Diamox) treatment (B), and after the addition of a sodium-glucose cotransporter-2 inhibitor (SGLT2i) (C).

\section{Reduction of fat tissue deposits in the lymphedematous limb}

The most astonishing and important finding of this case is that the SGLT2i greatly reduced the size of the lymphedematous limb. Adding the SGLT2i to the initial acetazolamide treatment was expected to simply facilitate the decongestion of the diseased limb. Although additional effects of SGLT2 $i$ were not impressive at the earlier observation period (day 30), the size of the lymphedematous limb was dramatically reduced at the later observation period (day 70). Adipose tissue deposits are present in lymphedematous limbs, $, 9,10$ and surgical liposuction effectively improves the lymphedematous status..$^{9,10}$ Accordingly, a pharmacologic effect of SGLT2i to improve chronic lymphedema could be expected on the basis of the well-known metabolic effect of SGLT2i to reduce body fat mass. ${ }^{11,12}$ Unfortunately, this potential effect of SGLT2 $i$ was not evaluated in the present study because the patient's lymphedema was not examined using a suitable imaging modality, such as computed tomography ${ }^{9}$ or magnetic resonance imaging. ${ }^{10}$

\section{Conclusions}

In conclusion, because the main pathologic constituents of lymphedema include excess fluid accumulation in the interstitial space $^{1}$ and adipose tissue deposition, ${ }^{9,10}$ a two-step pharmacologic approach of administering acetazolamide as well as an SGLT2i, as reported here, could be expected to be an efficient treatment regimen for some lymphedematous patients. In particular, SGLT2i may have yet unknown pleiotropic effects for modulating adipose tissue proliferation in lymphedema. ${ }^{9,10}$ Future studies are required to investigate the indication, effects, and mechanisms of this pharmacologic therapy for improving lymphedema, incorporating the use of lymphological-specific examinations, e.g., lymphography, lymphoscintigraphy, or MRI lymphangiography. ${ }^{1}$

\section{References}

1. The diagnosis and treatment of peripheral lymphedema. 2009 consensus document of the international society of lymphology. Lymphology 2009;42:5160.

2. Kataoka H. The "chloride theory", a unifying hypothesis for renal handling and body fluid distribution in heart fail- 
ure pathophysiology. Med Hypotheses 2017;104:170-3.

3. Kataoka H. Treatment of hypochloremia with acetazolamide in an advanced heart failure patient and importance of monitoring urinary electrolytes. J Cardiol Cases 2018;17:80-4.

4. Kataoka H. Acetazolamide as a potent chloride-regaining diuretic: short- and long-term effects, and its pharmacologic role under the 'chloride theory' for heart failure pathophysiology. Heart Vessels 2019;34:1952-60.

5. Kataoka H. Biochemical determinants of changes in plasma volume after decongestion therapy for worsening heart failure. J Cardiac Fail 2019;25: 213-7.

6. Kataoka H. Comparison of changes in plasma volume and renal function between acetazolamide and conventional diuretics: understanding the mechanical differences according to the "chloride theory". Cardiology 2020;145:21523.
7. Masuda T, Watanabe Y, Fukuda K, et al. Unmasking a sustained negative effect of SGLT2 inhibition on body fluid volume in the rat. Am J Physiol Renal Physiol 2018;315:F653-64.

8. Kataoka H, Yoshida Y. Enhancement of the serum chloride concentration by administration of sodium-glucose cotransporter- 2 inhibitor and its mechanisms and clinical significance in type 2 diabetic patients: a pilot study. Diabetol Metab Syndr 2020;12:5.

9. Brorson H, Ohlin K, Olsson G, et al. Adipose tissue dominates chronic arm lymphedema following breast cancer: an analysis using volume rendered CT images. Lymphat Res Biol 2006;4:199210.

10. Hoffner M, Peterson $P$, Månsson S, et al. Lymphedema leads to fat deposition in muscle and decreased muscle/water volume after liposuction: a magnetic resonance imaging study. Lymphat Res Biol 2018;16:174-81.

11. Bolinder J, Ljunggren Ö, Johansson L, et al. Dapagliflozin maintains glycaemic control while reducing weight ant body fat mass over 2 years in patients with type 2 diabetes mellitus inadequately controlled on metformin. Diabetes Obes Metab 2014;16:159-69.

12. Sugiyama S, Jinnouchi H, Kurinami N, et al. Dapagliflozin reduces fat mass without affecting muscle mass in type 2 diabetes. J Atheroscler Thromb 2018;25:467-76.

13. Trayes KP, Studdiford JS, Pickle S, et al. Edema: diagnosis and management. Am Fam Physician 2013;88:102-10.

14. Hallow KM, Helmlinger G, Greasly PJ, et al. Why do SGLT2 inhibitors reduce heart failure hospitalization?: a differential volume regulation hypothesis. Diabetes Obes Metab 2018;20:479-87.

15. Ohara K, Masuda T, Murakami T, et al. Effects of the sodium-glucose cotransporter 2 inhibitor dapagliflozin on fluid distribution: a comparison study with furosemide and tolvaptan. Nephrology 2019;24:904-11. 